\title{
In vivo characterization of chronic traumatic encephalopathy using [F-18]FDDNP PET brain imaging
}

\author{
Jorge R. Barrio ${ }^{a, 1}$, Gary W. Small ${ }^{b}$, Koon-Pong Wonga, Sung-Cheng Huang ${ }^{a}$, Jie Liu (刘捷) ${ }^{a}$, David A. Merrill ${ }^{b}$, \\ Christopher C. Giza', Robert P. Fitzsimmons ${ }^{\mathrm{d}}$, Bennet Omalu' ${ }^{\mathrm{e}}$, Julian Bailes ${ }^{\mathrm{f}}$, and Vladimir Kepe ${ }^{\mathrm{a}, 1}$
}

Departments of ${ }^{a}$ Molecular and Medical Pharmacology, ${ }^{b}$ Psychiatry and Biobehavioral Sciences and the Semel Institute for Neuroscience and Human Behavior, and ' Neurosurgery and Pediatrics, The David Geffen School of Medicine, University of California, Los Angeles, CA 90095; ${ }^{\mathrm{C}}$ Fitzsimmons Law Offices, Wheeling, WV 26003; 'Department of Medical Pathology and Laboratory Medicine, School of Medicine, University of California, Davis, Sacramento, CA 95817; and ' Department of Neurosurgery, NorthShore University Health System and University of Chicago Pritzker School of Medicine, Evanston, IL 60201

Edited by Marcus E. Raichle, Washington University in St. Louis, St. Louis, MO, and approved January 9, 2015 (received for review May 29, 2014)

Chronic traumatic encephalopathy (CTE) is an acquired primary tauopathy with a variety of cognitive, behavioral, and motor symptoms linked to cumulative brain damage sustained from single, episodic, or repetitive traumatic brain injury (TBI). No definitive clinical diagnosis for this condition exists. In this work, we used [F-18]FDDNP PET to detect brain patterns of neuropathology distribution in retired professional American football players with suspected CTE $(n=14)$ and compared results with those of cognitively intact controls $(n=28)$ and patients with Alzheimer's dementia (AD) $(n=24)$, a disease that has been cognitively associated with CTE. [F-18]FDDNP PET imaging results in the retired players suggested the presence of neuropathological patterns consistent with models of concussion wherein brainstem white matter tracts undergo early axonal damage and cumulative axonal injuries along subcortical, limbic, and cortical brain circuitries supporting mood, emotions, and behavior. This deposition pattern is distinctively different from the progressive pattern of neuropathology [paired helical filament (PHF)-tau and amyloid- $\beta$ ] in AD, which typically begins in the medial temporal lobe progressing along the cortical default mode network, with no or minimal involvement of subcortical structures. This particular [F-18]FDDNP PET imaging pattern in cases of suspected CTE also is primarily consistent with PHF-tau distribution observed at autopsy in subjects with a history of mild TBI and autopsy-confirmed diagnosis of CTE.

traumatic brain injury | chronic traumatic encephalopathy

[F-18]FDDNP PET | tau imaging | concussions

$T^{1}$ he consensus statement on concussions from the Fourth International Conference on Concussion in Sports (Zurich 2012) (1) defines acute mild traumatic brain injury (mTBI) or cerebral concussion as a brain injury with a complex pathophysiological process induced by biomechanical forces. Cerebral concussion causes white matter axonal injury due to axonal shearing and stretching (2), typically resulting in the rapid onset of short-lived impairment of neurological function that resolves spontaneously and largely reflects a functional disturbance rather than a structural injury. As such, no abnormality is seen on standard structural neuroimaging determinations (1).

A number of early literature reports described a neurodegenerative disease associated with a history of repetitive TBI in retired professional boxers $(3,4)$, with a prevalence rate of up to $47 \%$ among retired professional boxers aged $50 \mathrm{y}$ and older who boxed for more than $10 \mathrm{y}$ (5). Initially named "punch drunk syndrome" (3) and dementia pugilistica (4), this syndrome is now known as chronic traumatic encephalopathy (CTE) in the current literature $(6,7)$.

Compelling autopsy evidence (6-8) and neurobehavioral determinations (9) of retired professional American football athletes indicate that a subgroup develops neurodegenerative and clinical changes typical of CTE, a progressive syndrome distinctively different from Alzheimer's disease (AD), which is the most common form of dementia in the elderly (10). The connection between multiple concussions and subconcussive head impacts (2) and CTE is compelling, because history of repetitive concussions is the strongest risk factor for development of CTE in numerous contact sports (e.g., American football, rugby, boxing, ice hockey, soccer, and professional wrestling), in war veterans with a history of blast or blunt force TBI, and in conditions where trauma to the head occurs for various reasons (e.g., falls during seizures, head-banging in autistic children, motor vehicle and domestic accidents, domestic violence and abuse) $(6,8,11-14)$. As with most neurodegenerative diseases, clinical diagnosis remains elusive due to the lack of specificity of CTE clinical symptomatology criteria, and histopathological examination of brain at autopsy is the most definitive diagnostic modality $(6,8,11)$.

The novel imaging approaches leading to the in vivo characterization of CTE brain neuropathology premortem (e.g., PET) are complementary to structural imaging modalities [e.g., diffusion tensor imaging MRI (DTI MRI)] and offer a specific and sensitive strategy to facilitate diagnosis of CTE. Neuronal and glial fibrillar hyperphosphorylated microtubule-associated protein tau deposits composed of paired helical filament (PHF)-tau are the primary brain proteinopathy of CTE based on autopsy determinations, and their $3 \mathrm{R} / 4 \mathrm{R}$ tau isoform ratio is similar to that of $\mathrm{AD}$ (11). Their topographically predictable pattern of distribution was used as a basis for a severity staging system of CTE neuropathology (7), ranging from mild (neuropathology

\section{Significance}

Mild traumatic brain injuries are frequent events in the general population and are associated with a severe neurodegenerative disease, chronic traumatic encephalopathy (CTE). This disease is characterized by abnormal accumulation of protein aggregates, primarily tau proteins, which accumulate in brain areas responsible for mood, fear, stress, and cognition. There is no definitive clinical diagnosis of CTE at the present time, and this new work shows how a tau-sensitive brain imaging agent, [F-18]FDDNP, may be able to detect the disease in living people with varying degrees of symptoms. Early detection would facilitate the most effective management strategies and provide a baseline to measure the effectiveness of treatments.

Author contributions: J.R.B., G.W.S., R.P.F., B.O., J.B., and V.K. designed research; J.R.B., G.W.S., J.L., D.A.M., C.C.G., and V.K. performed research; B.O. contributed new reagents/ analytic tools; J.R.B., K.-P.W., S.-C.H., and V.K. analyzed data; and J.R.B. and V.K. wrote the paper.

Conflict of interest statement: J.R.B., G.W.S., and S.-C.H. are coinventors of the [F-18]FDDNP PET technology that is covered under University of California, Los Angeles patents and licensed to TauMark, LLC. J.R.B., G.W.S., R.P.F., and B.O. have a financial interest in TauMark, LLC.

This article is a PNAS Direct Submission.

${ }^{1}$ To whom correspondence may be addressed. Email: jbarrio@mednet.ucla.edu or vkepe@ mednet.ucla.edu.

This article contains supporting information online at www.pnas.org/lookup/suppl/doi:10. 1073/pnas.1409952112/-/DCSupplemental. 
stages I and II) to advanced (neuropathology stages III and IV) (7) (Tables S1 and S2). In addition, more than $80 \%$ of analyzed pathologically confirmed CTE cases also show transactive response (TAR) DNA-binding protein of $\sim 43 \mathrm{kDa}$ (TDP-43) either as inclusions in sparse neurites in cortex, medial temporal lobe structures, and brainstem in CTE neuropathology stages I-III, as widespread neuronal and glial inclusions in severe CTE cases (neuropathology stage IV), or in CTE cases with motor neuron disease $(7,15)$ (Tables S1 and S2). CTE cases also can exhibit the presence of other fibrillar protein aggregates. McKee et al. (7) and Omalu et al. (8) reported that in autopsy determinations, less than half of all CTE cases and less than one third of "pure" CTE cases show amyloid- $\beta(\mathrm{A} \beta)$ deposits, predominantly as scattered cortical diffuse plaques in low density (Tables S1 and S2). Of note is that subjects with A $\beta$ deposits were significantly older than those without. Moreover, their neuropathology was more severe than that in cases without $\mathrm{A} \beta$ deposits and was often combined with $\alpha$-synuclein deposits (7). As an example, as reported by McKee et al. (7), of 30 CTE cases with at least some cortical A $\beta$ deposits (of 68 confirmed CTE cases), 29 brains were from subjects who died in their seventh decade of life and one from a subject who died in his sixth decade.

Subsequent to our preliminary report (16), in this work we use [F-18]FDDNP, an imaging agent for fibrillar insoluble protein aggregates (16-20), and PET imaging with the aim of establishing $(i)$ topographic brain localization of [F-18]FDDNP PET signals indicative of fibrillar neuroaggregates in retired professional American football players with suspected CTE (mTBI group) vs. controls (CTRL); (ii) determination of [F-18]FDDNP PET signal patterns in the mTBI group; (iii) presence of [F-18]FDDNP PET signal as a measure of neuropathology in the brain areas involved in mood disorders related neurocircuits; (iv) correlation of [F-18]FDDNP PET results with neuropathology distributions in confirmed CTE cases; $(v)$ differential patterns of [F-18]FDDNP PET signals, and thus deposition of fibrillar neuroaggregates, in the mTBI group with respect to the AD group; and (vi) preliminary demonstration of differences in [F-18]FDDNP PET signal patterns in mTBI cases with different etiology, i.e., contact-sport-related mTBI in retired professional American football players vs. blast-induced mTBI in war veterans. We further intended to demonstrate that tau (vs. $A \beta$ ) specificity of high affinity PET molecular imaging probes may not be a necessary requirement when used in CTE subjects with primary proteinop- athy in the form of PHF-tau (8): PET imaging probes potentially sensitive to TDP-43 aggregates and $A \beta$ deposits, which are present in higher densities almost exclusively in older CTE cases with more advanced neuropathology (e.g., stage IV), could better define disease progression based on quantification of differences in regional loads of combined neuropathologies because additional neuropathologies appear in predictable topographical and temporal patterns.

\section{Results}

Brain [F-18]FDDNP PET Patterns in Suspected CTE (mTBI Group). In all types of neurodegenerative proteinopathies, including CTE, pathology-based diagnosis using protein specific immunohistochemistry (IHC) does not result only from the presence of protein pathology in the brain, but rather from detection of disease specific topographic patterns of pathology distribution combined with quantitation of regional and global pathology loads. Molecular imaging agents targeting such fibrillar protein pathologies must therefore accurately and reliably detect these diseasespecific patterns of distribution and their changes both in the pattern complexity and in regional pathology loads that may evolve due to the progressive nature of these neurodegenerative diseases.

Demographic data for all mTBI group subjects are provided in Table 1. We identified four distinctive topographical patterns of brain [F-18]FDDNP PET signal in the mTBI group, which are presented in increasing complexity from $\mathrm{T} 1$ to $\mathrm{T} 4$ in Fig. 1 (Upper) in three views (transaxial, top row; sagittal, middle row; and coronal, bottom row). All four patterns distinguish the mTBI subjects from cognitively intact control subjects (CTRL group) (Fig. 1, Upper, and Table 2).

The observed [F-18]FDDNP PET signal patterns are defined as follows:

i) Pattern $\mathrm{T} 1$ is predominantly subcortical in brainstem (midbrain) with localized involvement of the limbic medial temporal lobe structures (limited to amygdala).

ii) Pattern T2 shows [F-18]FDDNP PET signal in all subcortical areas analyzed in this study, in all limbic medial temporal lobe areas [amygdala and medial temporal lobe (MTL); hippocampus, entorhinal cortex, parahippocampal gyrus)], and in parts of the frontal cortex including anterior cingulate gyrus (ACG).

iii) Pattern T3 shows further increases in signal intensity and pattern complexity: all affected areas in the T2 pattern plus

Table 1. Demographic information for the mTBI group

\begin{tabular}{|c|c|c|c|c|c|c|c|c|c|c|}
\hline Subject & Age (y) & Education (y) & Race & $\begin{array}{c}\text { Active } \\
\text { career }(y)\end{array}$ & Position & Retirement (y) & Diagnosis & $\begin{array}{l}\text { MMSE } \\
\text { (score) }\end{array}$ & $\begin{array}{l}\text { HAM-D } \\
\text { score }\end{array}$ & $\begin{array}{l}\text { HAM-A } \\
\text { score }\end{array}$ \\
\hline TBI01 & 59 & 18 & AA & 16 & Linebacker & 28 & MCI-MD & 25 & 11 & 13 \\
\hline TBI02 & 64 & 17 & $\mathrm{C}$ & 10 & Quarterback & 38 & Normal & 30 & 5 & 6 \\
\hline TBI03 & 73 & 18 & $\mathrm{C}$ & 16 & Offensive guard & 44 & Dementia & 17 & 8 & 5 \\
\hline TBI04 & 50 & 15 & $\mathrm{C}$ & 14 & Defensive tackle & 20 & MCl-MD & 28 & 17 & 12 \\
\hline TBI05 & 45 & 18 & $\mathrm{C}$ & 12 & Center & 16 & $\mathrm{MCl}-\mathrm{A}$ & 29 & 6 & 0 \\
\hline TBI06 & 86 & 16 & $\mathrm{C}$ & 11 & Running back & 58 & $\mathrm{MCl}-\mathrm{A}$ & 27 & 3 & 3 \\
\hline TBI07 & 62 & 16 & $\mathrm{C}$ & 17 & Offensive guard & 28 & MCl-NA & 27 & 16 & 19 \\
\hline TBI08 & 51 & 18 & AA & 14 & Defensive lineman & 22 & $\mathrm{MCl}-\mathrm{MD}$ & 30 & 17 & 31 \\
\hline TBI09 & 59 & 15 & AA & 16 & Running back & 26 & MCl-MD & 27 & 14 & 12 \\
\hline TBI10 & 54 & 14 & AA & 12 & Wide receiver & 22 & MCl-MD & 25 & 15 & 16 \\
\hline TBI11 & 40 & 16 & $\mathrm{C}$ & 17 & Center & 7 & $\mathrm{MCl}-\mathrm{A}$ & 27 & 17 & 21 \\
\hline TBI12 & 54 & 16 & $\mathrm{C}$ & 16 & Offensive lineman & 20 & $\mathrm{MCl}-\mathrm{A}$ & 26 & 0 & 1 \\
\hline TBI13 & 62 & 16 & AA & 13 & Offensive lineman & 32 & $\mathrm{MCl}-\mathrm{A}$ & 28 & 33 & 30 \\
\hline TBI14 & 54 & 16 & C & 9 & Nose tackles & 25 & MCl-MD & 26 & 21 & 22 \\
\hline
\end{tabular}

A, amnestic; AA, African American; C, Caucasian; HAM-A, Hamilton Anxiety Score (mild to moderate symptoms: 18-24; moderate to severe: > 24); HAM-D, Hamilton Depression Score (mild symptoms: 8-16; moderate to severe: >16); MCI, mild cognitive impairment; MD, multiple domains; MMSE, mini-mental state examination (dementia range $<24$ ); NA, nonamnestic. 

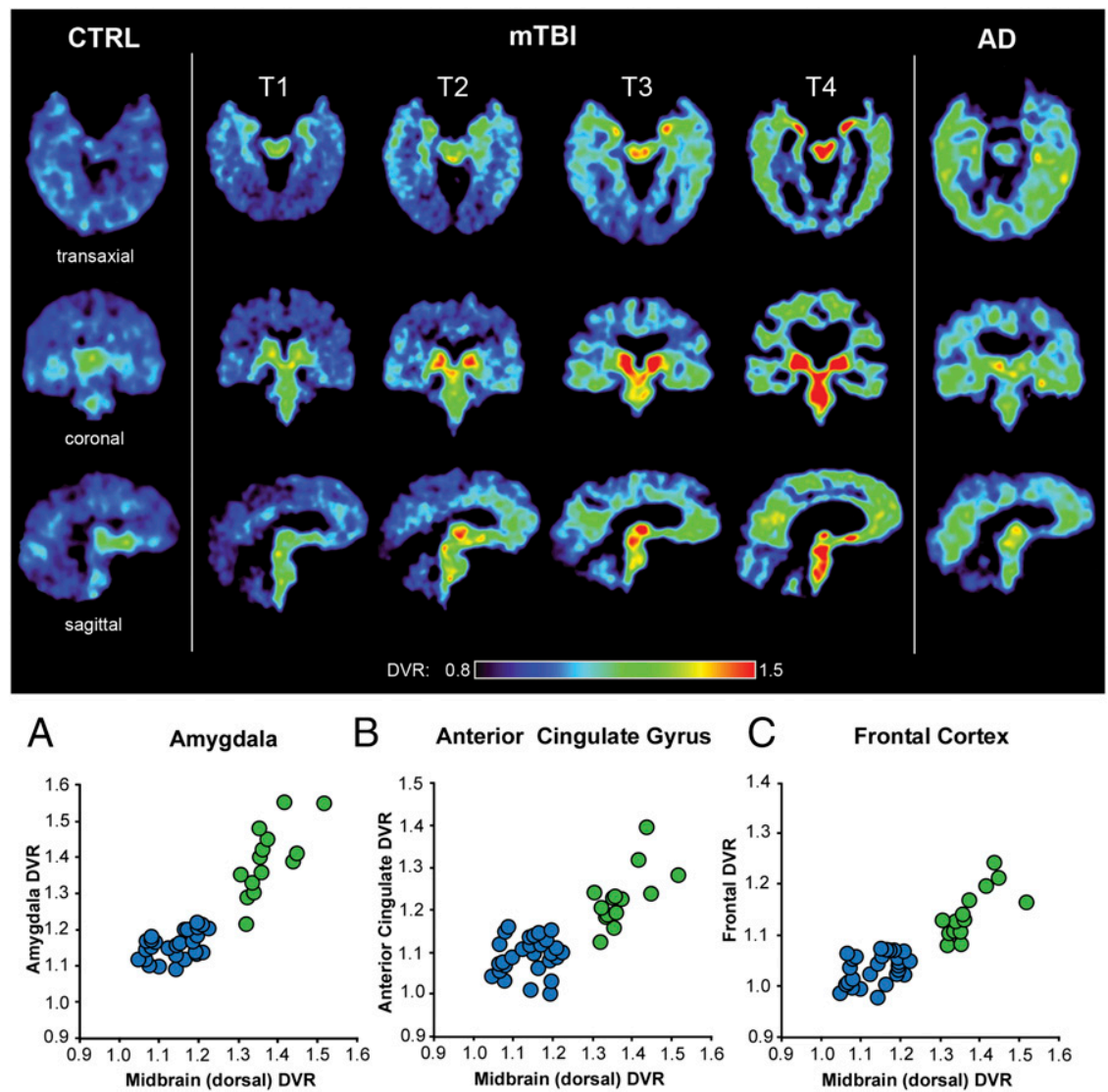

Fig. 1. (Upper) [F-18]FDDNP distribution volume ratios (DVR) parametric images showing patterns T1 to T4 of increased [F-18]FDDNP signal observed in the mTBI group compared with cognitive control subjects (Left). The T1 pattern shows involvement of two core areas which have consistently increased [F-18]FDDNP signal in all four patterns: amygdala (limbic) and dorsal midbrain (subcortical). Patterns T2 to T4 are marked by increase of [F-18]FDDNP signal in these two core regions and progressively larger number of subcortical, limbic, and cortical areas. Although more complex patterns (e.g., T4) overlap with $A D$ in the cortex, midbrain and amygdala signals are elevated above the levels in $A D$ (Table 2). An AD case is shown in the right column for comparison. (Lower) $A$ is a 2D scatter plot showing [F-18]FDDNP DVR values in two core areas consistently involved in CTE (subcortical structures (dorsal midbrain) and limbic structures (amygdala)), clearly demonstrating separation of $\mathrm{MTBI}$ and CTRL groups. $B$ and $C$ demonstrate similar separation effect when dorsal midbrain is compared with cortical areas typically associated with CTE and its mood disorders, namely anterior cingulate gyrus (ACG) (B) and frontal lobe (C). mTBI subjects are represented by green circles, and CTRL subjects are represented by blue circles. See SI Materials and Methods for additional correlations of [F-18]FDDNP PET DVR values in dorsal midbrain and amygdala with several cortical and subcortical areas (Fig. S1).

additional cortical areas [posterior cingulate gyrus (PCG), lateral temporal lobe (LTL), and parietal lobe]; this pattern is not associated with severe ventricular enlargement and prominent cortical atrophy commonly observed in aged retired boxers with dementia pugilistica.

iv) Pattern T4 shows high [F-18]FDDNP PET signal throughout the cortical, subcortical, and limbic medial temporal lobe structures, as well as in the white matter areas; this pattern was associated with significant brain atrophy (MRI or CT); possible comorbidity of CTE with other neurodegenerative diseases may be suspected, e.g., $\mathrm{AD}$ or end stage CTE progressing to, and simulating, AD.

All mTBI subjects $(n=14)$ were treated as a single group for further group comparison with the CTRL group $(n=28)$. Analysis of [F-18]FDDNP PET signal patterns T1-T4 identified the dorsal midbrain and amygdala as two core regions with consistently increased [F-18]FDDNP PET signals, which separated the mTBI group from the CTRL group as shown in the 2D correlation graph in Fig. $1 A$ (Lower). Statistical analysis (ANOVA with post hoc Tukey-Kramer test; Table 2) shows a high degree of statistical significance $(P<0.0001)$ for group separation in both areas and for a positive correlation between these two areas within the mTBI group (Spearman rank corre- lation coefficient: $r_{S}=0.745, P<0.01$; Table S3). All subcortical and limbic medial temporal lobe areas have also met strict criterion for good separation of mTBI and CTRL groups (TukeyKramer test at $P<0.0001$ ) (Table 2). Other subcortical areas also show positive Spearman rank correlations with both core regions (Fig. S1 and Table S3) reflective of similar trends of increasing regional [F-18]FDDNP distribution volume ratio (DVR) values with increasing [F-18]FDDNP PET signal pattern complexity from T1 to T4. However, some subcortical structures like thalamus and caudate/putamen in mTBI subjects show increased DVR values only in more severe cases.

In contrast to subcortical regions, cortical areas showed different trajectories of involvement in the [F-18]FDDNP PET signal patterns $\mathrm{T} 1-\mathrm{T} 4$ with a clear anterior-posterior gradient. This difference in cortical trajectories is reflected in separation of the mTBI and CTRL groups only in the ACG (Fig. $1 B$, Lower, and Fig. S1 $A$ ) and frontal lobe (Figs. $1 C$, Lower, and $2 A, z$ axis, and Table 2). Spearman rank correlations within the mTBI group were determined for combinations of all analyzed regions that have shown mTBI-CTRL group separations (and involvement in the [F-18]FDDNP PET signal patterns T1-T4) and correlations of the core regions (amygdala and dorsal midbrain) with a large number of subcortical regions [brainstem (pons), diencephalon (thalamus), and basal ganglia (striatum)] 
Table 2. [F-18]FDDNP DVR group mean values for mTBI group (14 subjects), control group (28 subjects), and Alzheimer's disease group (24 subjects)

\begin{tabular}{|c|c|c|c|c|c|c|c|c|c|c|c|c|c|c|}
\hline & \multicolumn{2}{|c|}{ Limbic } & \multicolumn{6}{|c|}{ Subcortical } & \multicolumn{6}{|c|}{ Cortical } \\
\hline & Amygd & MTL & Midb-V & Midb-D & Нypo-Th & Th & Pons & Str & $F$ & ACG & $P$ & PCG & LTL & OCC \\
\hline mTBI & 1.397 & 1.183 & 1.330 & 1.373 & 1.429 & 1.507 & 1.319 & 1.531 & 1.144 & 1.230 & 1.095 & 1.160 & 1.122 & 0.997 \\
\hline \multirow[t]{2}{*}{ CTRL } & 1.162 & 1.112 & 1.135 & 1.138 & 1.234 & 1.250 & 1.156 & 1.322 & 1.039 & 1.093 & 1.054 & 1.082 & 1.064 & 1.021 \\
\hline & $(0.036)$ & $(0.023)$ & $(0.057)$ & $(0.055)$ & $(0.049)$ & $(0.086)$ & $(0.064)$ & $(0.069)$ & $(0.033)$ & $(0.043)$ & $(0.026)$ & $(0.040)$ & $(0.026)$ & $(0.048)$ \\
\hline$A D$ & 1.242 & 1.189 & 1.137 & 1.164 & 1.229 & 1.318 & 1.145 & 1.345 & 1.112 & 1.141 & 1.148 & 1.180 & 1.155 & 1.059 \\
\hline$F$ value & $59.82^{*}$ & $73.26^{*}$ & $29.26^{*}$ & $26.56^{*}$ & $24.36^{*}$ & $15.01 *$ & $13.97^{*}$ & $21.82^{*}$ & $45.83^{*}$ & $13.72^{*}$ & $72.00 *$ & $44.93 *$ & $82.04^{*}$ & 5.43 \\
\hline \multicolumn{15}{|c|}{ Student $t$ test } \\
\hline mTBI-CTRL & * & * & * & * & * & * & * & * & * & * & * & * & * & * \\
\hline mTBI-AD & * & NS & * & * & * & * & * & * & NS & NS & NS & NS & NS & NS \\
\hline AD-CTRL & * & * & NS & NS & NS & NS & NS & NS & * & NS & * & * & * & NS \\
\hline \multicolumn{15}{|c|}{ Tukey-Kramer test } \\
\hline
\end{tabular}

Mean group DVR values (SD values given in parentheses). ACG, anterior cingulate gyrus; Amygd, amygdala; $F$, frontal; Hypo-Th, hypothalamus; LTL, lateral temporal lobe; Midb-D, dorsal midbrain; Midb-V, ventral midbrain; MTL, medial temporal lobe; NS, not significant $(P \geq 0.0001)$; Occ, occipital; $P$, parietal; PCG, posterior cingulate gyrus; Str, striatum (caudate nucleus and putamen); Th, thalamus (medial thalamus). ${ }^{*} P<0.0001 ;$ NS, not significant $(P \geq 0.0001)$.

and cortical regions (frontal, ACG, LTL, parietal) were all found to be significant $(P<0.05$; Table S3). It is noteworthy that these structures-brainstem (midbrain, pons), limbic medial temporal lobe (amygdala), and frontal lobe (frontal cortex, ACG) - are interconnected by brain circuitry supporting normal mood and behavioral functioning (21), which is perturbed early in subjects with a history of multiple concussions $(2,22)$.
Results of ANOVA statistical tests for separation of DVR values in all three groups are given for all areas analyzed, and areas that meet the Tukey-Kramer test criterion of $P<0.0001$ are identified for comparisons of the mTBI group with the CTRL and the AD groups separately (Table 2). Tables S4 (mTBI group), S5 (CTRL group), and S6 (AD group) show all regional DVR values for all subjects in all groups. Tables S7
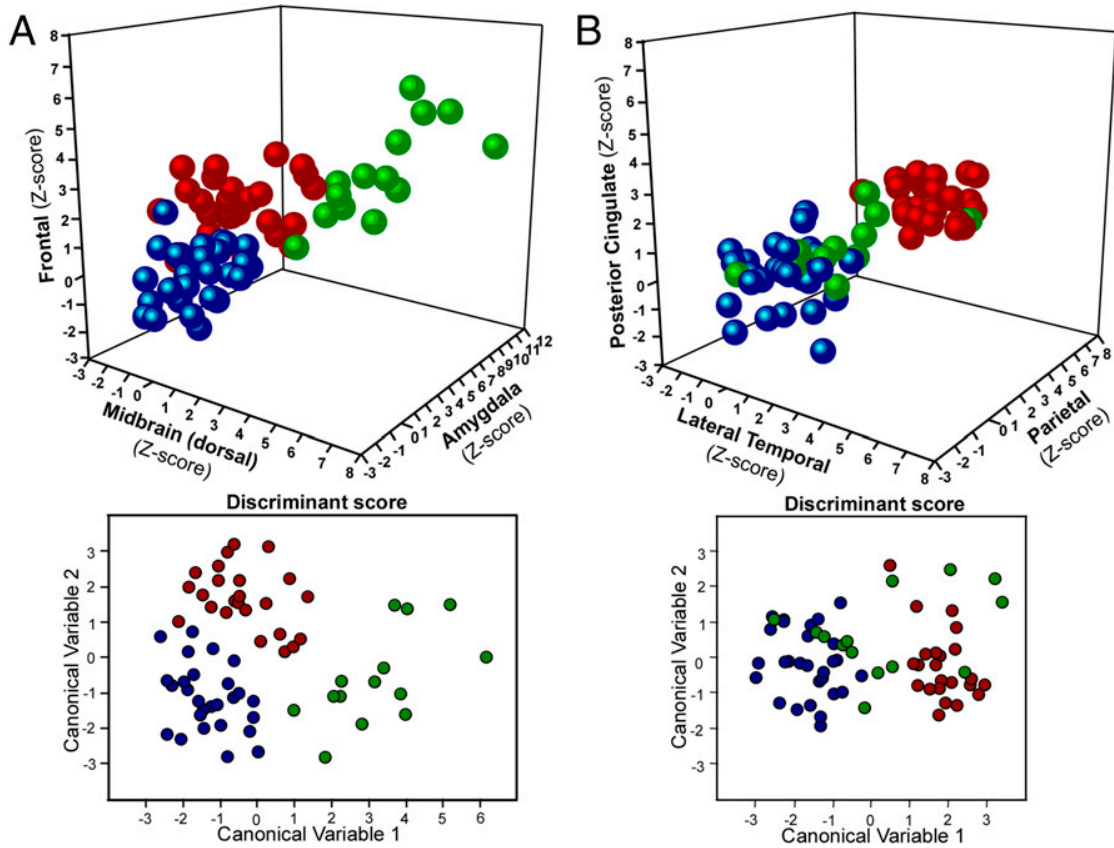

Fig. 2. [F-18]FDDNP PET DVR value analysis separates mTBI, CTRL and AD groups. (A) A 3D scatter plot correlation of subcortical regions (dorsal midbrain) with limbic structures (amygdala) and cortical regions (frontal lobe) shows that all three groups (mTBI, green; AD, red; CTRL, blue) can be effectively separated based on the differences in binding patterns in these three areas. The mTBI group is significantly separated from control group in all three areas and from the $A D$ group in the limbic and subcortical areas (Table 2). (B) A 3D scatter plot correlation of cortical structures alone, without comparison with subcortical or limbic structures, demonstrates that the mTBI group overlaps both with AD and CTRL groups. Results of discriminant analysis for the correlation of three areas depicted in each 3D scatter plot are shown underneath each graph. 
(mTBI group), S8 (CTRL group), and S9 (AD group) show all regional Z-score values for all subjects in all groups.

[F-18]FDDNP Results: Correlation with Autopsy of Confirmed CTE Cases. [F-18]FDDNP PET signal patterns T1-T4 are consistent with pathology reports showing involvement of subcortical structures (e.g., locus coeruleus, substantia nigra, dorsal raphe, thalamus, basal ganglia), limbic medial temporal lobe structures (amygdala and MTL), and frontal cortex $(6-8,10,11,23)$. The F-18] FDDNP PET signal in the subcortical structures is in accordance with the examples of tau IHC labeling of pons, midbrain, thalamus, and basal ganglia in CTE shown on large brain tissue samples in refs. 7, 10, 11, and 23 (Fig. S2, Left). Presence of tau IHC in subcortical regions is further supported by our evidence from confirmed CTE cases that were not scanned with [F-18]FDDNP PET (Fig. $3 I I$ and Fig. S2, Right). Midbrain and amygdala are unique brain regions with elevated [F-18]FDDNP signals found in all 14 mTBI subjects. DVR values shown in Table S4 suggest that the PET signal is not uniformly distributed throughout all midbrain structures. A dorsal-ventral gradient observed with PET imaging agrees well with tau IHC results (Fig. 3II, Left), which shows high IHC density in the periaqueductal gray in dorsal midbrain and lesser IHC levels in substantia nigra, red nucleus, and other nuclei situated in the ventral midbrain (Fig. S2, bottom example in the left panel) (11). Similarly, high [F-18]FDDNP signals in the amygdala and other limbic medial temporal lobe structures coincide with common CTE autopsy observations of high involvement of amygdala and MTL shown in deceased retired professional American football players (Fig. 3III) (7, 23).

[F-18]FDDNP PET in mTBI vs. AD. From a perspective of clinical diagnosis, CTE can be mistaken for AD, particularly at later stages (24). However, the pattern of subcortical and limbic medial temporal lobe (amygdala vs. MTL) [F-18]FDDNP signal distribution in $\mathrm{AD}$ is quite different from that of concussionbased mTBI (suspected CTE) (Figs. 1 and 2 and Fig. S1) (25). To

\section{Biomechanics of concussion II. Amygdala and Dorsal Midbrain: core regions in [F-18]FDDNP PET}

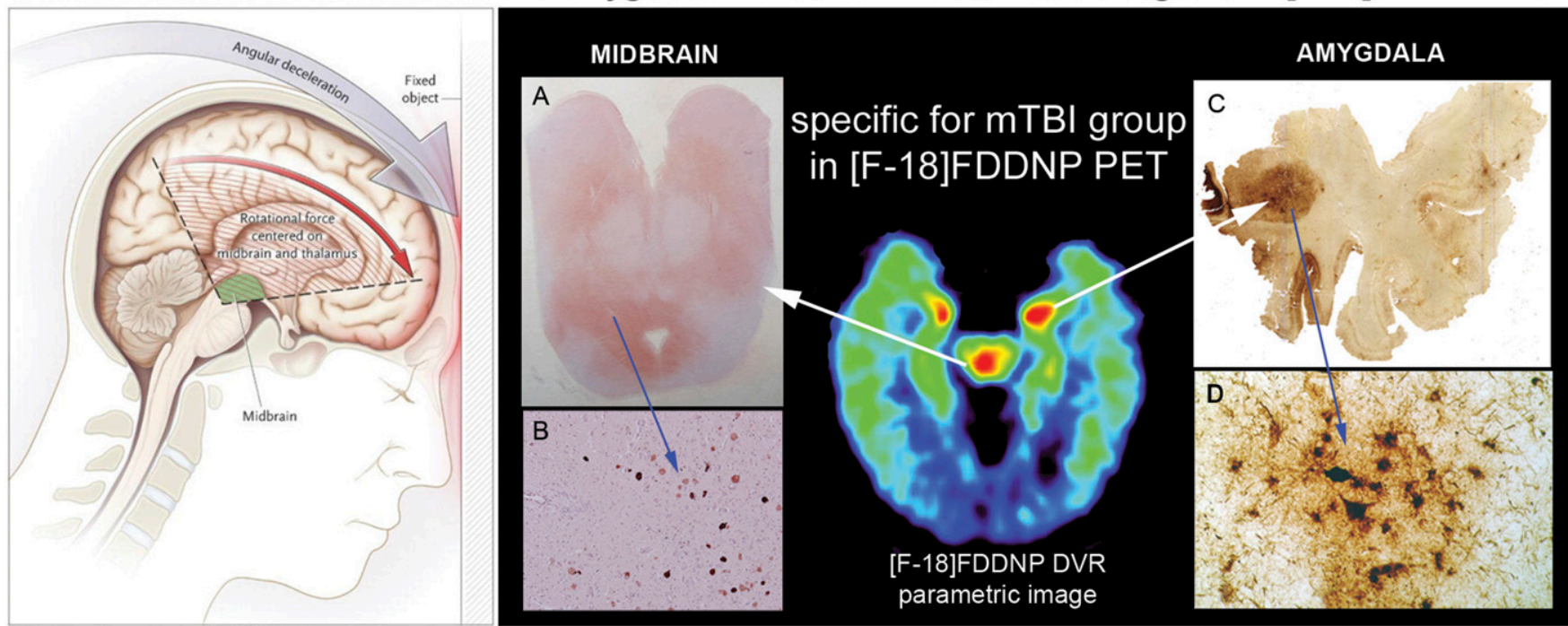

III. Robust tau IHC in amygdala and MTL is consistently present in symptomatic retired footbal players

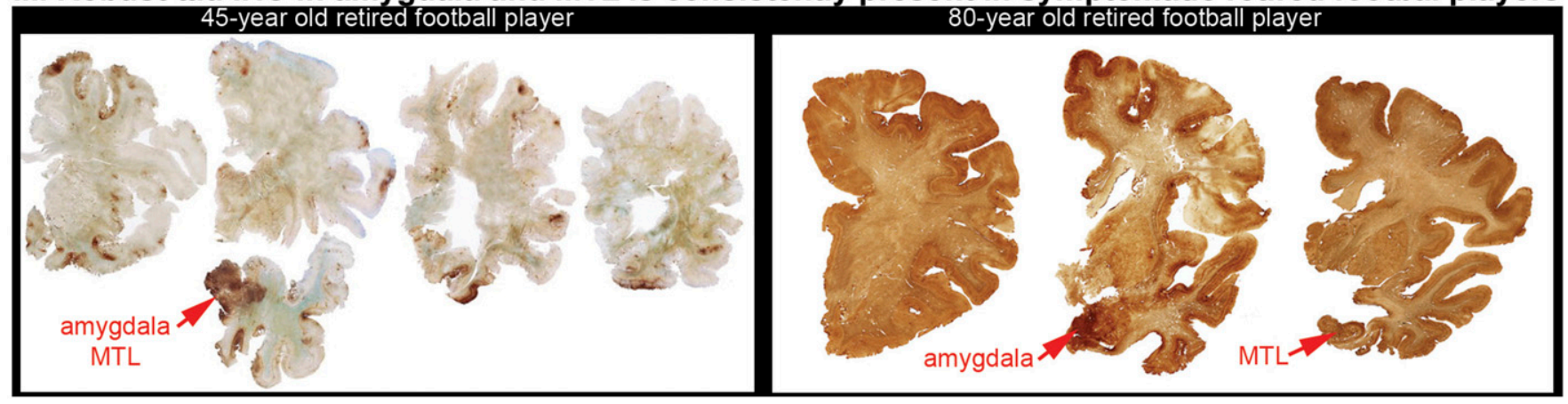

Fig. 3. Involvement of amygdala and midbrain areas in concussion-based mTBI is supported by both mechanistic concept of injury ( $/$ ) and by the results of neuropathological examinations in deceased retired American football players with premortem complaints of functional impairments (II and III). (I) Rotation of the brain in the sagittal plane during a concussion, associated with significant accelerations and deceleration, will have significant negative effect on the brain tissue in the midbrain and thalamus (green shaded area) and on the affected cortical areas (red area). Stretching, compression, and shearing of axons during such sudden brain movements is hypothesized to be the cause of axonal injury (reprinted from ref. 33; reproduced with permission from Massachusetts Medical Society.) Online version of ref. 33 also contains an animated version of this figure (www.nejm.org/doi/full/10.1056/NEJMcp064645). Similarly, rotation in the coronal plane has been shown to lead to consistent damage to midbrain region tracts (27). (II) $A-D$ show results of tau immunohistochemistry and demonstrate that in the mTBI group areas of increased [F-18]FDDNP signal in amygdala and dorsal midbrain coincide with presence of dense tau deposits in periaqueductal gray (PAG) in dorsal midbrain ( $A$ and $B$ ) and in amygdala ( $C$ and $D$; reprinted from ref. 21; reproduced with permission from Wolters Kluwer Health). (III) Amygdala and MTL areas are affected in the brains of retired professional American football players who died due to suicide (Left; 45 -y-old retired player; reprinted from ref. 21; reproduced with permission from Wolters Kluwer Health) or due to natural causes [Right; 80-y-old retired NFL player; (C) Oxford University Press (brain.oxfordjournals.org/content/136/1/43) (reprinted from ref. 11)]. Amygdala and MTL areas are the first areas with high density of tau deposits in the neocortex and remain one of the most affected cortical regions in the majority of retired professional American football player cases. 
facilitate visual comparison of AD and mTBI groups, we converted all DVR values in these two groups to Z-scores defined as Z-score $=\left(\mathrm{DVR}_{(\mathrm{ROI}-\mathrm{X})}-\mathrm{DVR}_{(\mathrm{CTR} \text { group-mean })}\right) / \mathrm{SD}_{(\mathrm{CTRLgroup})}$. CTRL group DVR mean values and SDs for each region of interest (ROI) are provided in Table 2. All graphs in Fig. 2 and Fig. S1 are shown on Z-score scales. Using the Tukey-Kramer test criterion of $P<0.0001$, it is quite clear that the mTBI group is separated from the $\mathrm{AD}$ group in the majority of subcortical areas and in amygdala but not in cortical areas, which is not surprising because $\mathrm{AD}$ is predominantly a cortical disease in terms of pathology accumulation. In the presence of significant cortical [F-18]FDDNP signals in both groups, our evidence shows that we can completely separate both groups using a combination of both core regions (amygdala and dorsal midbrain; $x$ axis and $y$ axis in the 3D graph in Fig. 2A).

The following general observations can be made:

i) The AD group showed [F-18]FDDNP DVR values similar to the CTRL group in subcortical regions (Table 2 and Fig. S1 $C-F)$. Both the CTRL and AD groups had increased variability in [F-18]FDDNP DVR values in subcortical regions compared with cortical and limbic areas (Table 2). In contrast to subcortical regions, the AD group showed significantly higher signals than did the CTRL in cortical areas, with the exception of occipital lobe and the ACG (Table 2 and Fig. 2B). The results were consistent with our previous reports on [F-18]FDDNP PET imaging in AD (18).

ii) The mTBI group showed higher DVR values compared with the AD group in all subcortical areas (Table 2 and Fig. $\mathrm{S} 1 C-F)$, which is most clearly observed in more complex [F-18]FDDNP PET signal patterns T2-T4. Similarly, amygdala (a limbic medial temporal lobe area) also demonstrated significantly higher involvement in the mTBI group than in the $\mathrm{AD}$ group, which is consistent with pathology observations in neuropathology stages III and IV (7). By contrast, other limbic medial temporal lobe structures (MTLs) showed DVR values in the mTBI group that were comparable to those in the $\mathrm{AD}$ group. Fig. $2 A$ presents a $3 \mathrm{D}$ scatter plot of $\mathrm{Z}$-scores with limbic medial temporal lobe structures (amygdala), subcortical (dorsal midbrain), and cortical structures (frontal) demonstrating a good separation of the mTBI group from the AD and CTRL groups. Fig. S1 illustrates similar correlative measures under the Tukey-Kramer test criterion of $P<$ 0.0001 involving subcortical, limbic, and selected cortical structures, which provided distinctive separation of the mTBI group from $\mathrm{AD}$ and CTRL groups.

iii) As previously reported (18), the AD group showed significantly higher [F-18]FDDNP signals in all cortical areas, and significant overlap with the mTBI group was observed in all cortical regions including MTL (Table 2, Fig. 2, and Fig. $\mathrm{S} 1 A$ ). As predicted from the clinical symptomatology (mood vs. cognitive), the mTBI group showed predominant involvement in the frontal and ACG ROIs compared with the AD group. Fig. $2 B$ further demonstrates that cortical [F-18]FDDNP PET signals do not discriminate between the mTBI and AD groups, whereas subcortical or limbic signals do. This lack of cortical discrimination becomes more obvious in the more severely affected and older (7) suspected CTE subjects (pattern T4).

These [F-18]FDDNP PET observations are consistent with the hypothesis that in individuals with suspected CTE, initial selected vulnerability is present in those circuits involving subcortical and limbic structures, including limbic structures in the medial temporal lobe. Through the medial temporal lobe and nodes in the prefrontal lobe (23), these circuits are connected with cortical areas involved in cognition via the default mode network (DMN), which includes the medial temporal lobe structures, the medial prefrontal cortex, posterior cingulate gyrus, and pre- cuneus and parietal cortex, which may provide a predictable pathway for tau progression in CTE through cortical areas. In AD, tau and $A \beta$ deposition are observed relatively early in the medial temporal lobe structures and the cascade of cortical pathology deposition typically follows the $\mathrm{DMN}$ with relatively lesser involvement of mood circuits in subcortical and limbic areas $(18,25,26)$, which provides the basis for differentiation of the mTBI and AD groups.

\section{Discussion}

Biomechanical Models of Concussions, Subject Population, and Brain Pathology. TBI has a profound medical and social impact, and it may occur under a variety of circumstances, from domestic accidents to war-related events. A concussive brain injury results from rapid rotational and translational accelerations and decelerations as well as impact decelerations, which exert significant forces on brain tissue and cause membrane and axonal injuries without overt gross bleeding or focal parenchymal damage (27). Following axonal and membrane cellular injury, isomorphic astrogliosis and microglial activation occur, which further interferes physically and chemically with regeneration and repair of disconnected axons (28), and leads to PHF-tau and TDP-43 deposition by a yet unknown mechanism. Because inflammation and white matter degeneration can persist for years (29), the brain is increasingly vulnerable to reinjury via traumatic axonal injury (30). Focal types of brain injury such as contusions or lacerations that commonly occur in moderate and severe traumatic injuries in humans or primate models of traumatic brain injury $(27,31,32)$ are only rarely reported in concussions (33).

As such, American football players present a relatively homogeneous group based on the type of concussions and subconcussions frequently present in this subject population (2). The initial, pioneering observation by Omalu et al. of extensive tauopathy deposition in the brains of American football players $(8,34)$, involving subcortical, limbic areas, and brain cortices, led to the unequivocal demonstration of CTE as a unique clinical entity (35). Most recent detailed studies by McKee et al. (7, 11, 21) provided confirmatory results of the unique pathological characteristics of CTE. In a study describing 85 brains including American football players, boxers, and veterans, among others, McKee et al. demonstrated the extensive tau aggregate distribution in subcortical and limbic structures, as well as cerebral cortices, in 68 brains with pathologically confirmed CTE in various stages of the disease (7).

In CTE cases resulting from playing American football, tau deposits consist of PHF-tau IHC positive neuronal neurofibrillary tangles (NFTs) and neuropil threads, as well as astrocytic tangles. Distribution of PHF-tau IHC positive structures follows a pattern that increases in complexity both with age and symptom severity $(6,7,11,21)$. In cases of CTE with advanced pathology (stages III and IV) (7), dense deposits of tau pathology were consistently found predominantly in subcortical structures and limbic areas of frontal and temporal lobes with extension to cortical areas. $\mathrm{A} \beta$ aggregates are not frequently a distinctive pathological feature of CTE, with less than $50 \%$ of the cases presenting predominantly scattered sparse deposition of cortical $A \beta$ in diffuse form, most frequently in later stages of pure CTE (stage IV) $(7,11)$. Often other protein aggregates, such as TDP-43 in more than $80 \%$ of all pathologically confirmed CTE cases, and more rarely $\alpha$-synuclein, can be present as secondary proteinopathies, also most prominently in later stages of the disease. TDP-43 is present in high density in midbrain of all CTE patients with motor neuron disease, but it is more variable in CTE patients stages I-III without motor neuron disease $(7,15$, 21) (for topographic details of different pathologies in various disease stages see Fig. S2 and Tables S1 and S2). Thus, it cannot be discounted that some of the [F-18]FDDNP signal in the brainstem, thalamus, and basal ganglia of the mTBI group, particularly in disease stages with more advanced pathology and 
increased deposit densities (stage IV) (7), originates from the presence of TDP-43. TDP-43 contains amino acid sequences that make it aggregation prone in vitro and also in vivo because a portion of neuronal and glial TDP-43 inclusion bodies in motor neuron disease and CTE appear as filamentous structures (36) but are generally not considered amyloid due to missing amyloid specific thioflavin-S fluorescent stain. Recent evidence shows that at least some of these inclusion bodies do show the typical thioflavin-S staining, which provides evidence for presence of amyloid fibrillar structures within them (36).

Brain-Specific Neuropathology Accumulation Measured with [F-18]FDDNP PET. This work offers compelling evidence of the ability of [F-18]FDDNP PET to detect neuropathology in the living brain of American football players in a manner consistent with the pattern of deposition found at autopsy $(6,7,34)$. [F-18]FDDNP has a high affinity for tau fibrils $\left(K_{\mathrm{D}}=36.7 \pm 11.6 \mathrm{nM}\right.$ vs. $K_{\mathrm{D}}=$ $5.52 \pm 1.97 \mathrm{nM}$ for $\mathrm{A} \beta 42$ fibrils) (37), which provides a high binding potential (BP; defined as $R / K_{\mathrm{D}}$; where $R=$ number of tau aggregate binding sites per volume of brain tissue) for tau imaging visualization in vivo (38). The very low regional density of other proteinaceous $\beta$-sheet-containing aggregates (e.g., TDP-43, diffuse $A \beta$ ) would result in a low [F-18]FDDNP binding potential for non-tau aggregates, below the limit of sensitivity of PET, in the earlier CTE stages (stages I and II) as previously shown with the low abundance of $\alpha$-synuclein in a Lewy body dementia patient (17). However, the ability of [F-18]FDDNP PET to detect A $\beta$ becomes important to help in the characterization of $\mathrm{AD}$ comorbidity when it is present, particularly in advanced neuropathology CTE stages (stage IV) when proteinaceous neuropathologies are widespread, and quantitation of total neuropathology load can reflect additional pathology burden due to comorbid conditions. This investigation further confirms the unique sensitivity of [F-18] FDDNP to tau aggregates in vivo, as demonstrated by earlier work in patients with a neurodegenerative tauopathy, progressive supranuclear palsy (PSP) (19). All these studies in humans are supported by the demonstration of the ability of the 6-dialkylamino naphthalenyl-2-cyanoacrylate scaffold (present in FDDNP) to bind tightly to tau aggregates as previously shown by X-ray analysis of crystals obtained by cocrystallization of DDNP with tau segments (39), in vitro binding affinities with tau fibrils, tau rodent models, and postmortem autopsy results and comparisons with premortem scans $(16,17)$.

These findings of brain tau accumulation and distribution in this subject population are also consistent with earlier observations that subcortical structures such as brainstem and thalamus are considered as the fulcrum or center point of force vectors that bear the maximal rotational forces in American football players receiving concussions (Fig. 3) (2, 33). Axonal degeneration due to thousands of cranial impacts resulting in concussive and subconcussive injuries leads to other neurodegenerative changes including PHF-tau deposition throughout the areas of selective vulnerability in heavily interconnected cortico-striatopallido-thalamic loops that support emotions, mood, and behavior (for a review of this topic, see ref. 22). Initial widespread involvement of the limbic areas of the medial temporal lobe (followed by the frontal lobe) is also observed in the subjects studied in this investigation and defines core regions that are consistently affected as neuropathology distribution increases in complexity during CTE evolution (Fig. 1, Upper).

These [F-18]FDDNP PET imaging patterns are observed in all cases studied thus far, suggesting a "fingerprint" PET scan of neuropathology accumulation that is characteristic of CTE. This [F-18]FDDNP PET imaging topographic distribution, moreover, is quite different from that found in $\mathrm{AD}$ (Fig. 2) and is consistent with profiles of neuropathology distribution described by Omalu et al. (6) and McKee et al. (7) in autopsy specimens with confirmed CTE. Omalu et al. defined four distinct pathology phe- notypes (6), of which phenotype III (brainstem predominant) closely resembles the T1 pattern, whereas phenotypes I and II fit the description of patterns T2 and T3 described in this work. Comparison with the pathology stages as defined by McKee et al. (7) clearly identifies that [F-18]FDDNP PET signal patterns T1-T4 may primarily result from more affected examples of neuropathology stage II and from stages III and IV (Table S2) $(7,11)$, as they all show prominent neuropathology in medial temporal lobe structures. [F-18]FDDNP PET signal pattern T4 parallels neuropathology of severe stage IV in widespread distribution of signal and in signs of severe atrophy of ventricles. Patterns T2 and T3 would fit into stage III and milder examples of stage IV (Table S2) (7).

[F-18]FDDNP Signal Is Consistent with Mood and Cognitive Disorders in Suspected CTE. Dysfunction within brain circuits, caused by axonal damage leading eventually to PHF-tau aggregate accumulation in CTE, is believed to be the trigger for the mood disorders observed in these subjects who have experienced multiple concussions and subconcussions. Presence of axonal injury in brainstem and cortical white matter has been demonstrated postmortem in teenagers who had a history of playing high school American football (11). Such neurodegenerative processes can produce typical manifestations, initially involving behavioral and mood symptoms and leading to subsequent impairments of cognition and, in some cases, motor impairments in later stages (22).

The [F-18]FDDNP PET results in this work show direct early involvement of brain areas that participate in processing of emotions, mood, and behavior. We observed neuropathology deposition using [F-18]FDDNP PET even in the minimally affected subjects, in the amygdala, several areas of the frontal cortex including the ACG, medial thalamus, hypothalamus, and dorsal midbrain (which contains the periaqueductal gray; Fig. 3). This midbrain pathology distribution pattern has also been reported recently in a retired Australian rugby player with CTE (stage IV) (11). Our [F-18]FDDNP PET imaging results, together with neuropathology observations at autopsy, highlight the selective vulnerability of these midbrain structures, some of which are involved in maintaining consciousness, modulating pain, and controlling defensive behavior (27).

Resting state fMRI studies also have shown that connectivity in these circuits is altered in generalized anxiety disorder (40), panic disorder (41), and depression (42). Some aspects of these neuronal circuits have also been closely connected with other neurodegenerative tauopathies, e.g., progressive supranuclear palsy, as demonstrated by Gardner et al. (43) and further confirmed by direct measurement of PHF-tau accumulation with [F-18]FDDNP PET (19). It has been speculated that in this case, the connectivity of these circuits offers an opportunity for transmissibility of tau aggregation to different brain regions in a predictable manner (44). It is possible that similar relationship between neuropathology accumulation and the pattern of involved neuronal circuits also exists in CTE, and our current observations seem to confirm this possibility.

Regardless of the predictable mechanism of neuropathology transmissibility through the connectivity of these circuits, the present study supports a correlation between the mechanical effects of concussions and subconcussions on specific brain areas and accumulation of neuroaggregates with the subsequent neuronal dysfunction and their corresponding neuropsychiatric consequences. However, effective confirmation awaits a larger cohort of subjects, also including subjects suffering brain concussions that may be biomechanically different from those found in American football players (e.g., war veterans, boxers). Exposure to blasts from explosive devices has caused changes consistent with TBI in war veterans, with clinical signs and changes in their DTI MRI scans consistent with traumatic diffuse axonal injury. Our initial [F-18]FDDNP PET studies in war veterans and other severe TBIs $(n=8)$ seem to indicate that blast-induced 
concussions present with a different neuropathology deposition profile than that found in blunt-force TBI or contact-sportrelated concussions (Fig. S3).

Clinical diagnosis of CTE remains elusive, and alternative approaches using blood-based biomarkers of cellular degeneration (45) are being explored; however, such methods may provide information about the presence of neurodegenerative processes but not their brain distribution and thus may be inferior to brain imaging biomarkers. DTI MRI (46) can provide useful information about axonal injury in the white matter in CTE but is not specific to this condition as other neurodegenerative diseases (e.g., AD) can have similar patterns of white matter degeneration caused by different mechanisms. Resting state fMRI has also been used to assess changes in large-scale cognitive networks in concussed athletes and in subjects with a history of TBI (47).

The present work suggests that, based on the definition of disease-specific tau pathology deposition stages in suspected CTE, we can use neuropathology deposition as a brain tissue target for PET molecular imaging probes with in vivo sensitivity for these neuroaggregates. However, considering that tau deposits are not specific for CTE but are present in other tauopathies and also in $\mathrm{AD}$, a simple positive or negative reading with PHF-tau PET imaging probes is in itself clinically irrelevant. Only when combined with the regional sensitivity of PET will these probes provide significant in vivo information about regional tau deposition throughout the entire brain, at the earliest possible stage when medical management is most likely to succeed with CTE. This potential can be realized if the molecular imaging probe used has sufficient sensitivity for in vivo detection of relevant neuropathology aggregates and reliably reflects the presence of these aggregates in all brain regions affected in a concentrationdependent manner, in agreement with autopsy determinations and with the prevalent mood and cognitive symptoms observed in this patient population.

\section{Conclusion}

This work with [F-18]FDDNP PET offers a sensitive method to visualize and quantify the regional presence of neuroaggregates in the living brain of human subjects with mTBI and suspected CTE and generates useful information about the mechanisms underlying disease staging and the mood disorders observed in these patients. It also offers the potential for early diagnosis of CTE in living subjects, when the probability of successful experimental therapeutic interventions would be greatest. Based on neuropathology indicators (7) (Tables S1 and S2), these [F-18]FDDNP distribution patterns in suspected CTE are largely due to PHF-tau in milder CTE stages (stages I-III), with possible TDP-43 and A $\beta$ contributions in advanced CTE stages (stage IV) and in CTE with comorbidities. These [F-18]FDDNP topographic distribution patterns are unique among neurodegenerative diseases and significantly different from those found in AD (Figs. 1 and 2), progressive supranuclear palsy (19), and frontotemporal lobar degeneration (20). Thus, [F-18]FDDNP PET offers critical help in their differential diagnosis within a context of clinical history, physical examination, neuropsychiatric evaluation, and conventional radiological scans. These promising results provide the basis for a larger trial to determine the scope of this imaging procedure for CTE and for the use of this PET imaging technique to monitor disease progression in follow-up studies.

\section{Materials and Methods}

Written, informed consent for this [F-18]FDDNP PET imaging study was obtained from subjects in accordance with procedures of the Office of the Human Research Protection Program (OHRPP) of the University of California, Los Angeles, under strict, standard ethics guidelines.

Patient Population and Neurobehavioral Symptoms. The mTBI group included subjects with an increased risk of developing CTE as a result of having re- ceived repetitive concussions and subconcussions and presenting persistent cognitive, behavioral, and psychiatric problems, as defined by McKee et al. (7). It consisted of 14 retired male professional athletes who played American football professionally with histories of mood and cognitive symptoms as described above and in Table 1. Subjects TBI01-TBI05 from a preliminary report (16) have been included in this work. All mTBI subjects were collegeeducated retired professional American football players (age range, 40-86 y mean $\pm S D=57.2 \pm 11.6 \mathrm{y}$; mean years of education $\pm S D=16.2 \pm 1.4 \mathrm{y}$ ) who had played from 9 to 17 combined preprofessional (high school, college) and professional years (mean $\pm \mathrm{SD}=13.4 \pm 3.6$ ). All players, with the exception of a 64-y-old former quarterback (TBI02; Table 1), showed evidence of cognitive impairment. A standard neuropsychological test battery indicated that 12 subjects had a diagnosis of mild cognitive impairment $(\mathrm{MCl})$, which is a risk state for dementia. A 73-y-old former offensive guard had a diagnosis of dementia (TBI03). Most subjects also showed symptoms of depression (mean \pm SD: HAM-D score $=12.8 \pm 8.3$ ) and anxiety (mean \pm SD: HAM-A score $=12.1 \pm$ 8.7). The $\mathrm{mTBI}$ subjects also had more prominent cognitive and mood symptoms than motor symptoms. As part of the neurological examinations, motor and balance performance were measured on nine subjects using a modified Balance Error Scoring System (BESS) assessment (mean score \pm SD $=18.9 \pm 5.6$ ) (48) and the Chronic Brain Injury Scale (mean score $\pm S D=2.9 \pm 1.1$ ) (49).

Radiosynthesis. [F-18]FDDNP was prepared as previously described (50) following current US Pharmacopeia 823 requirements for chemistry, manufacture, and control of PET radiopharmaceuticals for human use (51).

PET Scanning. All PET scans of the mTBI group were performed on a Biograph PET/CT camera, except for one subject who was scanned using an ECAT HR+ PET camera (subject TBI01) (both Siemens/CTI), with subjects in the supine position and with the imaging plane parallel to the orbito-meatal line as described previously (18). A bolus of [F-18]FDDNP (320-550 MBq) was injected through an indwelling venous catheter as a bolus i.v. injection following a 10-min attenuation scan. The dynamic data acquisition was started at the time of injection, and the following time frames were collected: $6 \times 30,4 \times 180$, and $10 \times 300 \mathrm{~s}$ (total duration: $65 \mathrm{~min}$ ). All PET scans were decay corrected and reconstructed using filtered back-projection (Hann filter, 5.5-mm FWHM) with scatter correction and measured attenuation correction. The resulting images from the PET/CT camera contained 109 contiguous slices with a plane-to-plane separation of $2.0 \mathrm{~mm}$. Performance of PET scanners (e.g., Biograph, ECAT HR or ECAT EXACT HR+ scanner; Siemens $(\mathrm{TI})$ was tested using the Hoffman brain phantom, and no significant differences between images from the two scanners were observed. All subjects from the $A D$ and CTRL groups were scanned as previously reported (18) and summarized in the SI Materials and Methods.

Imaging Data Analysis. Quantification of the [F-18]FDDNP binding data for the $\mathrm{mTBI}$ group was performed using the Logan graphical method, with the cerebellar gray matter as the reference region, for time points between 15 and $65 \mathrm{~min}(52,53)$. The relative distribution volume (DVR) parametric images were generated and analyzed with the use of regions of interest (ROIs) drawn bilaterally on the coregistered MRI or CT scans for a number of cortical, limbic, and subcortical areas as defined below. No atrophy correction of the PET data was performed. Partial volume effects result in reduction of the PET signal due to brain atrophy, and if performed, this correction would increase the intensity of the [F-18]FDDNP brain signal even further. Results of [F-18]FDDNP PET data quantification are provided as DVR values (Tables S4-S6) and as Z-scores (Tables S7-S9). DVR values for all ROIs are also provided in cumulative form (as group mean DVR value \pm SD) for 28 cognitively intact control subjects and for 24 AD subjects previously reported (18). These subjects were included in the analysis to provide negative (CTRL group) and positive (AD group) reference points for low and high DVR values in the areas where fibrillar protein aggregates are present in $A D$ (predominantly cortical areas). Both the CTRL and AD groups appear as relatively homogeneous groups based on age and cognitive performance, but the TBI group contains subjects with a range of impairments.

ROIs. ROI sets included subcortical areas [striatum (Str), medial thalamus (Th), hypothalamus (Hypo-Th), dorsal midbrain (Midb-D), ventral midbrain (Midb-V), pons (Pons)], limbic areas of the medial temporal lobe [amygdala (Amygd) hippocampus with parahippocampal gyrus and entorhinal cortex (MTL)], and cortical areas [frontal lobe (F), anterior cingulate gyrus (ACG), parietal lobe (P), posterior cingulate gyrus (PCG), lateral temporal lobe (LTL) and occipital lobe (Occ)]. ROls were drawn bilaterally on each region, with the exception of the dorsal midbrain (Midb-D), where only one ROI was placed, and striatum (Str), which has been determined as an average of caudate nucleus and putamen. 
MRI Scanning. MRI scans were performed for the purpose of anatomical reference to aid the analysis of PET data as described previously (18). T1weighted magnetization-prepared rapid acquisition gradient-echo (MPRAGE) MRI volumetric scans, using a 3-T Siemens Allegra MRI scanner, were obtained for nine $\mathrm{mTBI}$ subjects (sagittal plane; repetition time, 2,300 ms; echo time $2.93 \mathrm{~ms} ; 160$ slices; slice thickness, $1 \mathrm{~mm}$; skip, $0.5 \mathrm{~mm}$; in-plane voxel size, $1.3 \times$ $1.3 \mathrm{~mm}$; field of view, $256 \times 256$; flip angle, $8^{\circ}$ ). Four of the other $\mathrm{mTBI}$ subjects received $C T$ scans because they could not tolerate an MRI scan (e.g., because of anxiety, claustrophobia, or metal in the body). One subject (TBI10) was unable to undergo either MRI or CT scans.

Statistical Analysis. Statistical analyses were performed with the use of SAS software (version 9.2). $P<0.0001$ was considered statistically significant unless otherwise stated. One-way ANOVA with Tukey-Kramer post hoc multiple comparisons was used to test for statistically significant differences in regional [F-18] FDDNP binding (DVR values) among the three groups of subjects. Multivariate ANOVA (MANOVA) was used to determine if a selected pair of regional DVR variables showed significant differences among normal controls and CTEs. Spearman rank correlations were computed between regional [F-18]FDDNP binding within the $\mathrm{mTBI}$ group to determine their associations, and scatter plots were generated to visualize how regional DVR variables related to one another $(P<0.05$ was considered statistically significant for this test). Discriminant analysis (DA) (54)

1. McCrory $\mathrm{P}$, et al. (2013) Consensus statement on concussion in sport: The 4th International Conference on Concussion in Sport held in Zurich, November 2012. Br J Sports Med 47(5):250-258.

2. Bailes JE, Petraglia AL, Omalu BI, Nauman E, Talavage T (2013) Role of subconcussion in repetitive mild traumatic brain injury. J Neurosurg 119(5):1235-1245.

3. Martland HS (1928) Punch drunk. JAMA 91(15):1103-1107.

4. Millspaugh JA (1937) Dementia pugilistica. U S Nav Med Bull 35(3):297-303.

5. Roberts AH (1969) Brain Damage in Boxers: A Study of the Prevalence of Traumatic Encephalopathy Among Ex-Professional Boxers (Pitman Medical \& Scientific Publishing Company, London).

6. Omalu B, et al. (2011) Emerging histomorphologic phenotypes of chronic traumatic encephalopathy in American athletes. Neurosurgery 69(1):173-183.

7. McKee AC, et al. (2013) The spectrum of disease in chronic traumatic encephalopathy. Brain 136(Pt 1):43-64

8. Omalu BI, et al. (2005) Chronic traumatic encephalopathy in a National Football League player. Neurosurgery 57(1):128-134, discussion 128-134.

9. Stern RA, et al. (2011) Long-term consequences of repetitive brain trauma: Chronic traumatic encephalopathy. PM R 3(10, Suppl 2):S460-S467.

10. Stein TD, Alvarez VE, McKee AC (2014) Chronic traumatic encephalopathy: A spectrum of neuropathological changes following repetitive brain trauma in athletes and military personnel. Alzheimers Res Ther 6(1):4

11. McKee AC, Daneshvar DH, Alvarez VE, Stein TD (2014) The neuropathology of sport Acta Neuropathol 127(1):29-51.

12. Omalu B, et al. (2011) Chronic traumatic encephalopathy in an Iraqi war veteran with posttraumatic stress disorder who committed suicide. Neurosurg Focus 31(5):E3, 10.3171/2011.9.FOCUS11178.

13. Geddes JF, Vowles GH, Robinson SF, Sutcliffe JC (1996) Neurofibrillary tangles, but not Alzheimer-type pathology, in a young boxer. Neuropathol Appl Neurobio/ 22(1):12-16.

14. Hof PR, Knabe R, Bovier P, Bouras C (1991) Neuropathological observations in a case of autism presenting with self-injury behavior. Acta Neuropathol 82(4):321-326.

15. McKee AC, et al. (2010) TDP-43 proteinopathy and motor neuron disease in chronic traumatic encephalopathy. J Neuropathol Exp Neurol 69(9):918-929.

16. Small GW, et al. (2013) PET scanning of brain tau in retired national football league players: Preliminary findings. Am J Geriatr Psychiatry 21(2):138-144.

17. Smid LM, et al. (2013) Postmortem 3-D brain hemisphere cortical tau and amyloid- $\beta$ pathology mapping and quantification as a validation method of neuropathology imaging. J Alzheimers Dis 36(2):261-274.

18. Small GW, et al. (2006) PET of brain amyloid and tau in mild cognitive impairment N Engl J Med 355(25):2652-2663.

19. Kepe V, et al. (2013) PET imaging of neuropathology in tauopathies: Progressive supranuclear palsy. J Alzheimers Dis 36(1):145-153.

20. Barrio JR, et al. (2009) Dissecting molecular mechanisms in the living brain of dementia patients. Acc Chem Res 42(7):842-850.

21. McKee AC, et al. (2009) Chronic traumatic encephalopathy in athletes: Progressiv tauopathy after repetitive head injury. I Neuropathol Exp Neurol 68(7):709-735.

22. Price JL, Drevets WC (2010) Neurocircuitry of mood disorders. Neuropsychopharmacology 35(1):192-216.

23. Stern RA, et al. (2013) Clinical presentation of chronic traumatic encephalopathy. Neurology 81(13):1122-1129.

24. Randolph C (2014) Is chronic traumatic encephalopathy a real disease? Curr Sports Med Rep 13(1):33-37.

25. Protas HD, et al. (2012) Prediction of cognitive decline based on hemispheric cortical surface maps of FDDNP PET. Neuroimage 61(4):749-760.

26. Braak H, Braak E (1991) Neuropathological stageing of Alzheimer-related changes. Acta Neuropathol 82(4):239-259.

27. Adams JH, Graham DI, Murray LS, Scott G (1982) Diffuse axonal injury due to nonmissile head injury in humans: an analysis of 45 cases. Ann Neurol 12(6):557-563. was used to evaluate the performance of classification of subjects with known group memberships (CTRL, $\mathrm{mTBI}$, or $A D$ ) based on the selected combinations of various regional [F-18]FDDNP DVR values as predictor variables. Because of a large number of possible combinations of predictor variables for DA, a backward elimination analysis was performed to determine the set of predictor variables that optimally discriminating the three groups (54). All predictor variables were first included, and the least significant variable ( $F$-test, $P>0.15$ ) was removed in a stepwise manner. This variable elimination procedure was repeated until no variable could be removed. The resulting reduced DA model functions and their canonical variables were used to assess the degree of overlapping between groups by calculating the percent of correct group classification and the overall classification accuracy, both of which were cross-validated by a leave-one-out method (54).

ACKNOWLEDGMENTS. We thank Natacha Donoghue and Jacqueline Martinez for subject scheduling and management; John Williams, University of California, Los Angeles, Nuclear Medicine Clinic, for performing the PET scans; and Shelley West, Billy West, and Bus Cook for guidance with subject referral and for their helpful input. Funding from the National Institutes of Health (P01-AG025831 and M01-RR00865), the Toulmin Foundation, and Robert and Marion Wilson is highly appreciated. No company provided research funding for this study.

28. Buss A, et al. (2004) Gradual loss of myelin and formation of an astrocytic scar during Wallerian degeneration in the human spinal cord. Brain 127(Pt 1):34-44.

29. Johnson VE, et al. (2013) Inflammation and white matter degeneration persist for years after a single traumatic brain injury. Brain 136(Pt 1):28-42.

30. Blaylock RL, Maroon J (2011) Immunoexcitotoxicity as a central mechanism in chronic traumatic encephalopathy-A unifying hypothesis. Surg Neurol Int 2:107.

31. Strich SJ, Oxon DM (1961) Shearing of nerve fibers as a cause of brain damage due to head injury: A pathological study of twenty cases. Lancet 278(7200):443-448.

32. Prins M, Greco T, Alexander D, Giza CC (2013) The pathophysiology of traumatic brain injury at a glance. Dis Model Mech 6(6):1307-1315.

33. Ropper AH, Gorson KC (2007) Clinical practice. Concussion. N Engl J Med 356(2):166-172.

34. Omalu BI, et al. (2006) Chronic traumatic encephalopathy in a national football league player: Part II. Neurosurgery 59(5):1086-1092, discussion 1092-1093.

35. Gavett BE, et al. (2011) Clinical appraisal of chronic traumatic encephalopathy: Current perspectives and future directions. Curr Opin Neurol 24(6):525-531.

36. Robinson JL, et al. (2013) TDP-43 skeins show properties of amyloid in a subset of ALS cases. Acta Neuropathol 125(1):121-131.

37. Harada R, et al. (2013) Comparison of the binding characteristics of [18F]THK-523 and other amyloid imaging tracers to Alzheimer's disease pathology. Eur J NuCl Med Mol Imaging 40(1):125-132.

38. Mintun MA, Raichle ME, Kilbourn MR, Wooten GF, Welch MJ (1984) A quantitative model for the in vivo assessment of drug binding sites with positron emission tomography. Ann Neurol 15(3):217-227.

39. Landau M, et al. (2011) Towards a pharmacophore for amyloid. PLoS Biol 9(6):e1001080.

40. Prater KE, Hosanagar A, Klumpp H, Angstadt M, Phan KL (2013) Aberrant amygdalafrontal cortex connectivity during perception of fearful faces and at rest in generalized social anxiety disorder. Depress Anxiety 30(3):234-241.

41. Pannekoek JN, et al. (2013) Aberrant limbic and salience network resting-state functional connectivity in panic disorder without comorbidity. J Affect Disord 145(1):29-35.

42. Ramasubbu $R$, et al. (2014) Reduced intrinsic connectivity of amygdala in adults with major depressive disorder. Front Psychiatry 5:17.

43. Gardner RC, et al. (2013) Intrinsic connectivity network disruption in progressive supranuclear palsy. Ann Neurol 73(5):603-616.

44. Clavaguera F, et al. (2013) Brain homogenates from human tauopathies induce tau inclusions in mouse brain. Proc Natl Acad Sci USA 110(23):9535-9540.

45. Jeter $C B$, et al. (2013) Biomarkers for the diagnosis and prognosis of mild traumatic brain injury/concussion. J Neurotrauma 30(8):657-670.

46. Shenton $\mathrm{ME}$, et al. (2012) A review of magnetic resonance imaging and diffusion tensor imaging findings in mild traumatic brain injury. Brain Imaging Behav 6(2):137-192.

47. Palacios EM, et al. (2013) Resting-state functional magnetic resonance imaging activity and connectivity and cognitive outcome in traumatic brain injury. JAMA Neurol 70(7):845-851.

48. Brown HJ, et al. (2014) Development and validation of an objective balance error scoring system. Med Sci Sports Exerc 46(8):1610-1616.

49. Jordan BD, et al. (1997) Apolipoprotein E epsilon4 associated with chronic traumatic brain injury in boxing. JAMA 278(2):136-140.

50. Liu J, et al. (2007) High-yield, automated radiosynthesis of 2-(1-6-[(2-[18F]fluoroethyl) (methyl)amino]-2-naphthylethylidene)malononitrile ([18F]FDDNP) ready for animal or human administration. Mol Imaging Bio/ 9(1):6-16.

51. US Pharmacopeial Convention (2013) Positron emission tomography drugs for compounding, investigational, and research uses. US Pharmacopeia 36-National Formulary 31 (US Pharmacopeial Convention, Rockville, MD), General Chapter 823.

52. Kepe V, Huang SC, Small GW, Satyamurthy N, Barrio JR (2006) Visualizing pathology deposits in the living brain of patients with Alzheimer's disease. Methods Enzymol 412:144-160.

53. Tantawy MN, et al. (2009) [(18)F]Fallypride dopamine D2 receptor studies using delayed microPET scans and a modified Logan plot. Nucl Med Biol 36(8):931-940.

54. McLachlan GJ (1992) Discriminant Analysis and Statistical Pattern Recognition (John Wiley \& Sons, Hoboken, NJ) 